\title{
Condition number in recovery of signals from partial fractional Fourier domain information
}

\author{
Figen S. Oktem ${ }^{1, *}$ and Haldun M. Ozaktas ${ }^{2}$ \\ ${ }^{1}$ Department of Electrical and Computer Engineering, University of Illinois at Urbana-Champaign, \\ Urbana, Illinois 61801, USA \\ ${ }^{2}$ Department of Electrical Engineering, Bilkent University, TR-06800 Bilkent, Ankara, Turkey
}

\begin{abstract}
The problem of estimating unknown signal samples from partial measurements in fractional Fourier domains arises in wave propagation. By using the condition number of the inverse problem as a measure of redundant information, we analyze the effect of the number of known samples and their distributions.
\end{abstract}

(c) 2013 Optical Society of America

\section{Introduction}

We consider a class of signal recovery problems where partial information in fractional Fourier domains is available and the aim is to estimate the unknown signal values. These problems can arise in optical, acoustical, and electromagnetic wave propagation because the propagation of waves can be considered as a process of continual fractional Fourier transformation, where the fractional order monotonically increases as a function of distance [1]. As the wave propagates, first the function itself, then its fractional Fourier transforms of increasing order, and then its Fourier transform (FT) is observed. The observation planes perpendicular to the axis of propagation correspond to fractional Fourier domains (FRFDs). Thus, the problem considered here corresponds to the problem of recovering waves from partial measurements distributed over several observation planes. Such problems can be encountered in many different circumstances. For instance, if measurements cannot be taken with sufficient spatial resolution, or if it is not possible to take measurements at certain parts of the field, then partial measurements can be taken at more than one plane for the purpose of recovering the missing samples. In this manner, one can compensate for the missing information arising from practical measurement constraints. The purpose of this work is not primarily to solve the resulting linear inverse problem numerically, but to develop insight into the nature of redundancy and information relationships in such problems for different instances of partial information. Further detail and references may be found in $[2,3]$.

\section{Problem Formulation}

The $a$ th-order fractional Fourier transform (FRT) [1] of a function $f(x)$, denoted by $f_{a}(x)$, is defined as

$$
f_{a}(x) \equiv \int_{-\infty}^{\infty} K_{a}\left(x, x^{\prime}\right) f\left(x^{\prime}\right) d x^{\prime}, \quad K_{a}\left(x, x^{\prime}\right) \equiv A_{\phi} e^{i \pi\left(\cot \phi x^{2}-2 \csc \phi x x^{\prime}+\cot \phi x^{\prime 2}\right)}, \quad A_{\phi}=\sqrt{1-i \cot \phi}, \quad \phi=a \pi / 2
$$

when $a \neq 2 k$ and $K_{a}\left(x, x^{\prime}\right)=\delta\left(x-x^{\prime}\right)$ when $a=4 k$ and $K_{a}\left(x, x^{\prime}\right)=\delta\left(x+x^{\prime}\right)$ when $a=4 k \pm 2$, where $k$ is an integer. The FRT operator $\mathscr{F}^{a}$ is additive in index: $\mathscr{F}^{a_{2}} \mathscr{F}^{a_{1}}=\mathscr{F}^{a_{2}+a_{1}}$ and reduces to the FT and identity operators for $a=1$ and $a=0$ respectively. The $a$ th order FRT transforms a signal to the oblique axis $x_{a}$ making angle $\phi=a \pi / 2$ with the $x$ axis, which is referred to as the $a$ th order FRT domain [1].

Consider two FRT domains of order $a_{1}, a_{2}$ such that each domain is sampled at $N$ uniform points. Let $\mathbf{f}=$ $[f(-N / 2), \ldots, f(N / 2-1)]^{T}$ and $\mathbf{g}=[g(-N / 2), \ldots, g(N / 2-1)]^{T}$ denote the vectors of length $N$ which represent the samples of the signals $f$ and $g$ in the $a_{1}$ th and $a_{2}$ th order FRT domains. If $a=a_{2}-a_{1}$, the relation between the signals at these domains is given by

$$
\mathbf{g}=\mathbf{F}^{\mathbf{a}} \mathbf{f}
$$

where $\mathbf{F}^{a}$ denotes the $N \times N$ ath order discrete FRT matrix given in [4].

Let $m_{1}$ and $m_{2}$ denote the number of known samples in the $a_{1}$ th and $a_{2}$ th order FRFDs. If the known indices in both domains form the vectors $\mathbf{k}=\left[k_{1}, \ldots, k_{m_{1}}\right]^{T}$ and $\mathbf{n}=\left[n_{1}, \ldots, n_{m_{2}}\right]^{T}$, then the vectors $f(\mathbf{k})=\left[f\left(k_{1}\right), \ldots, f\left(k_{m_{1}}\right)\right]^{T}$ 
and $g(\mathbf{n})=\left[g\left(n_{1}\right), \ldots, g\left(n_{m_{2}}\right)\right]^{T}$ contain the known signal values of $f$ and $g$, respectively. Similarly, if the unknowns have indices $\overline{\mathbf{k}}$ and $\overline{\mathbf{n}}$, then $f(\overline{\mathbf{k}})$ and $g(\overline{\mathbf{n}})$ represent the unknown signal values.

Let $\mathbf{F}^{\mathbf{a}}(\mathbf{n}, \mathbf{k})$ be an $m_{2} \times m_{1}$ submatrix of $\mathbf{F}^{a}$ obtained by choosing its $n_{1}$ th, $\ldots, n_{m_{2}}$ th rows and $k_{1}$ th, .., $k_{m_{1}}$ th columns. By choosing the same rows and the remaining columns, one can also construct the submatrix $\mathbf{F}^{\mathbf{a}}(\mathbf{n}, \overline{\mathbf{k}})$, which is $m_{2} \times\left(N-m_{1}\right)$. Then, the relation in (2) can be rewritten as

$$
g(\mathbf{n})=\mathbf{F}^{\mathbf{a}}(\mathbf{n}, \overline{\mathbf{k}}) f(\overline{\mathbf{k}})+\mathbf{F}^{\mathbf{a}}(\mathbf{n}, \mathbf{k}) f(\mathbf{k}) .
$$

Since only $f(\overline{\mathbf{k}})$ is unknown and required to be estimated in the above equation, the linear system of equations for the solution of the inverse problem is

$$
\mathbf{g}^{\prime}=\mathbf{F}^{\mathbf{a}}(\mathbf{n}, \overline{\mathbf{k}}) f(\overline{\mathbf{k}})
$$

where $\mathbf{g}^{\prime}=g(\mathbf{n})-\mathbf{F}^{\mathbf{a}}(\mathbf{n}, \mathbf{k}) f(\mathbf{k})$. Thus, in order to estimate $f(\overline{\mathbf{k}})$ which contains the unknown signal values of $f$, we need to solve the above system of equations. Similarly, to find $g(\overline{\mathbf{n}})$ which contains the unknown signal values of $g$, we can solve $\mathbf{f}^{\prime}=\mathbf{F}^{-\mathbf{a}}(\mathbf{k}, \overline{\mathbf{n}}) g(\overline{\mathbf{n}})$, where $\mathbf{f}^{\prime}=f(\mathbf{k})-\mathbf{F}^{-\mathbf{a}}(\mathbf{k}, \mathbf{n}) g(\mathbf{n})$. On the other hand, knowing the signal completely in one domain is equivalent to knowing it in all domains. Thus, it is enough to estimate the signal either in the $a_{1}$ th or $a_{2}$ th domain. We refer to the domain where to estimate the signal as the reference plane. In this work, we choose the domain with the largest number of known samples as the reference plane. That is, if $m_{1}>m_{2}$, the reference plane is chosen as the $a_{1}$ th order FRFD and otherwise, it is chosen as the $a_{2}$ th order FRFD.

We will investigate how the condition number of $\mathbf{F}^{\mathbf{a}}(\mathbf{n}, \overline{\mathbf{k}})$ or $\mathbf{F}^{-\mathbf{a}}(\mathbf{k}, \overline{\mathbf{n}})$ is affected by the distribution and number of known samples. We use the ratio of the largest singular value of the matrix to the smallest as the condition number [5]: $\operatorname{cond}(\mathbf{A})=\sigma_{\max }(\mathbf{A}) / \sigma_{\min }(\mathbf{A})$ which is always $\geq 1$. Values close to 1 indicate a well-conditioned matrix and small uncertainty in the solution. We use the condition number as a measure of redundant information in given samples since it measures how accurately the unknown samples can be estimated from the given samples.

We note that by using the unitary and symmetric properties of the matrix $\mathbf{F}^{a}$, one can easily show that changing the roles of the two domains does not affect the condition number. This supports the symmetrical structure of the problem and shows as expected that the direction of propagation does not create any difference on the solution.

\section{Numerical Results}

The fractional order $a$ is varied over $[0,1]$ with step 0.1 and the number of samples $N$ is chosen as 256 . We take $m_{1}$ and $m_{2}$, the number of known samples in the two domains, as powers of 2 up to 128 and then choose their symmetric values with respect to 128 up to 256. For different $m_{1}$ and $m_{2}$ pairs and different distributions of these known samples, the change in the logarithm of the condition number (to base 10) is investigated as a function of the order $a$. Since the spread of light increases with distance of propagation, increase in the order $a$ means a point in one domain will affect (interact with) more points in the other domain.

We considered both uniformly spread distributions of the known samples and distributions in which the knowns are accumulated at one end. The uniform distribution corresponds to the case when the measurement device has insufficient resolution. The accumulated distribution corresponds to the case when we can take measurements only over a limited interval. For both the uniform and accumulated distributions, we chose the known sample locations in the two domains either complementing each other or overlapping with each other. These variations illustrate the best case and worst case scenarios, and are shown in Fig. 1a.

In the first experiment, total number of known samples is equal to $N$, i.e. $m_{1}+m_{2}=N$. Figures $1 \mathrm{~b}$ and $1 \mathrm{c}$ show condition number vs. $a$ curves for uniform and accumulated distributions for the complementary case. The curves for all distributions when $m_{1}=16, m_{2}=240$ are plotted together in figure $1 \mathrm{~d}$.

As seen in Figures $1 \mathrm{~b}$ and $1 \mathrm{c}$, as the number of known samples $m_{1}$ and $m_{2}$ in the two domains get closer to each other, the condition number increases for all distributions. This indicates that distributing the $N$ known samples equally to the two domains causes the largest amount of redundant information in the given data.

As clearly seen from figure 1d, uniform distributions give better condition numbers than accumulated distributions. That is, measurements spread all over the plane carry more information than the same number of measurements concentrated in a particular region. When we compare the complementary and overlapping cases, we observe that while the complementary case is superior for small values of $a$, for larger values of $a$ they give similar results. The reason for this is that when the domains are close to each other, each point is interacting with small number of samples in the other domain, namely the region of interaction of each known point in the other domain is small; therefore, most of the points lying in these regions of interaction are unknown samples, so that there is not too much redundancy. However, as the value of $a$ is increased, the regions of interaction become larger and, a comparable number of known 


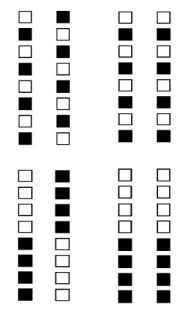

(a)

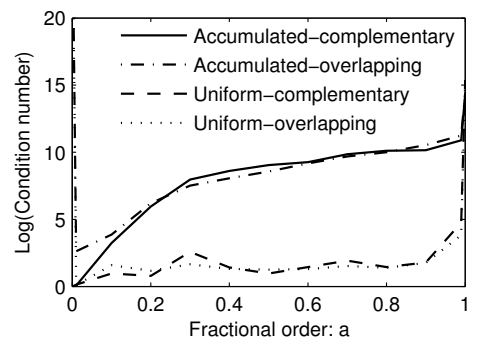

(d)

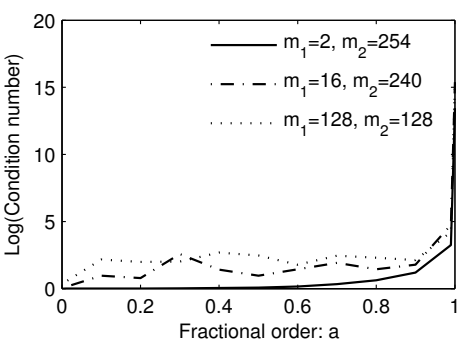

(b)

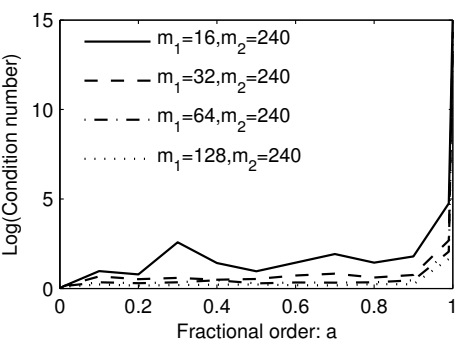

(e)

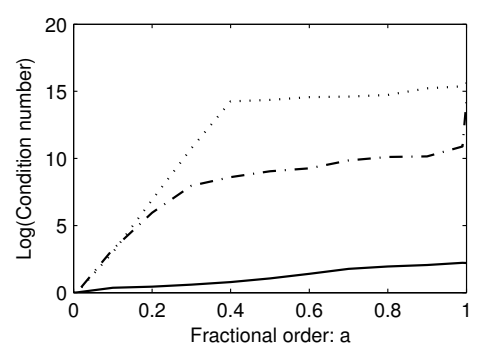

(c)

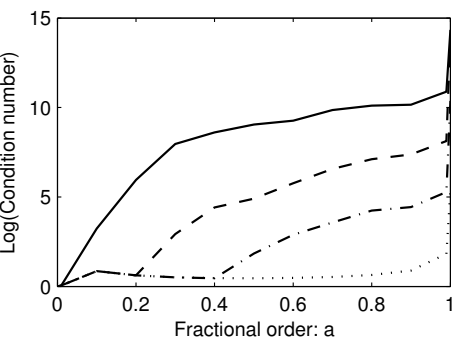

(f)

Fig. 1: (a) Illustration of different distributions: uniform-complementary (top-left), uniform-overlapping (top-right), accumulated-complementary (bottom-left), accumulated-overlapping (bottom-right). (b)-(c) Condition number vs $a$ for different pairs of $m_{1}$ and $m_{2}$ satisfying $m_{1}+m_{2}=N$, for the uniform-complementary and accumulatedcomplementary cases, respectively (the legend is valid for both plots). (d) Condition number vs $a$ for all distributions when $m_{1}=16$ and $m_{2}=240$. (e)-(f) Condition number vs $a$ for different pairs of $m_{1}$ and $m_{2}$ satisfying $m_{1}+m_{2}>N$, for uniform-complementary and accumulated-complementary cases, respectively (the legend is valid for both plots).

sample points fall into these regions in the complementary and overlapping cases, causing a comparable degree of redundancy in both cases. Thus we conclude that for values of $a$ that are not small, shifting the measurements in the transverse direction has little effect on its information content.

In the next experiment, we investigate the improvement in the condition number when $m_{1}+m_{2}>N$; in other words, when we increase the total number of known samples beyond $N$. Figures $1 \mathrm{e}$ and $1 \mathrm{f}$ are obtained by starting with the $m_{1}=16, m_{2}=240$ case and doubling $m_{1}$ each time when the known samples are distributed in uniform-complementary and accumulated-complementary fashion. As we increase the number of known samples in one domain, the condition numbers improve and thus the information to be used for the recovery of the signal improves. The improvement is more dramatic in the accumulated case since the condition numbers there were much larger to begin with, and less pronounced in the uniform case since the condition numbers there were already not very large.

H. M. Ozaktas was supported in part by the Turkish Academy of Sciences.

\section{References}

1. H. M. Ozaktas, Z. Zalevsky, and M. A. Kutay, The Fractional Fourier Transform with Applications in Optics and Signal Processing (New York: Wiley, 2001).

2. F. S. Oktem, "Signal representation and recovery under partial information, redundancy, and generalized finite extent constraints," Master's thesis, Bilkent Univ., Turkey (2009).

3. F. S. Öktem and H. M. Özaktaş, "Kesirli Fourier bölge aradeğerlemenin doğrusal cebirsel analizi," in IEEE 17th Signal Processing and Communications Applications Conference, pp. 872-875. 9-11 April 2009, Antalya, Turkey. (In Turkish.)

4. C. Candan, M. Kutay, and H. Ozaktas, "The discrete fractional fourier transform," IEEE Trans. Signal Process. 48, 1329-1337 (2000).

5. M. T. Heath, Scientific Computing: An Introductory Survey (New York: Mc Graw Hill, 2002). 